\title{
Global structure of one-sign solutions for a simply supported beam equation
}

Dongliang Yan ${ }^{1}$, Ruyun $\mathrm{Ma}^{1 *}$ and Xiaoxiao Su${ }^{1}$

"Correspondence:

mary@nwnu.edu.cn

${ }^{1}$ Department of Mathematics,

Northwest Normal University,

Lanzhou, P.R. China

\begin{abstract}
In this paper, we consider the nonlinear eigenvalue problem

$$
\begin{aligned}
& u^{\prime \prime \prime \prime}=\lambda h(t) f(u), \quad 0<t<1, \\
& u(0)=u(1)=u^{\prime \prime}(0)=u^{\prime \prime}(1)=0,
\end{aligned}
$$

where $h \in C([0,1],(0, \infty)) ; f \in C(\mathbb{R}, \mathbb{R})$ and $s f(s)>0$ for $s \neq 0$, and $f_{0}=f_{\infty}=0$, $f_{0}=\lim _{|s| \rightarrow 0} f(s) / s, f_{\infty}=\lim _{|s| \rightarrow \infty} f(s) / s$. We investigate the global structure of one-sign solutions by using bifurcation techniques.
\end{abstract}

MSC: 34B27; 34C23; 74K10

Keywords: Connected component; Green function; One-sign solutions; Bifurcation; Simply supported beam

\section{Introduction}

The deformations of an elastic beam whose both end-points are simply supported are described by the fourth order problem

$$
\begin{aligned}
& u^{\prime \prime \prime \prime}=\lambda h(t) f(u), \quad 0<t<1, \\
& u(0)=u(1)=u^{\prime \prime}(0)=u^{\prime \prime}(1)=0,
\end{aligned}
$$

where $h \in C([0,1],(0, \infty)) ; f \in C(\mathbb{R}, \mathbb{R})$ and $s f(s)>0$ for $s \neq 0$.

Existence and multiplicity of positive solutions of (1.1) have been extensively studied by several authors, see [1, 2, 5-10,13]. Cabada and Enguiça [2] developed the method of lower and upper solutions to show the existence and multiplicity of solutions, Jiang [6] and $\mathrm{Li}$ [7] proved the existence and multiplicity of solutions via the fixed point theorem in cone.

Bonanno and Di Bella [1] used variational method to obtain the following.

(c) The Author(s) 2020. This article is licensed under a Creative Commons Attribution 4.0 International License, which permits use, sharing, adaptation, distribution and reproduction in any medium or format, as long as you give appropriate credit to the original author(s) and the source, provide a link to the Creative Commons licence, and indicate if changes were made. The images or other third party material in this article are included in the article's Creative Commons licence, unless indicated otherwise in a credit line to the material. If material is not included in the article's Creative Commons licence and your intended use is not permitted by statutory regulation or exceeds the permitted use, you will need to obtain permission directly from the copyright holder. To view a copy of this licence, visit http://creativecommons.org/licenses/by/4.0/. 
Theorem A ([1, Theorem 1.1]) Let $f: \mathbb{R} \rightarrow \mathbb{R}$ be a continuous function. Assume that $x f(x)>0$ for all $x \neq 0$ and

$$
f_{0}=\lim _{|s| \rightarrow 0} f(s) / s=0, \quad f_{\infty}=\lim _{|s| \rightarrow \infty} f(s) / s=0 .
$$

Then, for every

$$
\lambda>\bar{\lambda}=:\left(\frac{8192}{27}+8 \pi^{2}\right) \max \left\{\inf _{d>0} \frac{d^{2}}{\int_{0}^{d} f(x) d x}, \inf _{d<0} \frac{d^{2}}{\int_{0}^{d} f(x) d x}\right\},
$$

the problem

$$
\begin{aligned}
& u^{\prime \prime \prime \prime}=\lambda f(u), \quad 0<t<1, \\
& u(0)=u(1)=u^{\prime \prime}(0)=u^{\prime \prime}(1)=0
\end{aligned}
$$

has at least four nontrivial classical solutions.

In the present work, we attempt to give a direct and complete description of the global structure of one-sign solutions of (1.1) under the assumptions:

(A1) $h:[0,1] \rightarrow(0, \infty)$ is continuous;

(A2) $f \in C(\mathbb{R}, \mathbb{R})$ and $s f(s)>0$ for $|s|>0$;

(A3) $f_{0}=0$;

(A4) $f_{\infty}=0$.

Let $Y=C[0,1]$ with the norm

$$
\|u\|_{\infty}=\max _{t \in[0,1]}|u(t)|
$$

We shall use Dancer's bifurcation theorem and some properties of superior limit of certain infinity collection of connected sets to establish the following.

Theorem 1.1 Let (A1), (A2), (A3), and (A4) hold. Then there exist a connected component $\mathcal{C}^{+} \subset \mathbb{R}^{+} \times C[0,1]$ of positive solutions of $(1.1)$ and a connected component $\mathcal{C}^{-} \subset \mathbb{R}^{+} \times$ $C[0,1]$ of negative solutions of $(1.1)$ such that

(1) $\mathcal{C}^{+}$is of $\subset$-shaped and joins $(+\infty, \mathbf{0})$ to $(+\infty, \infty)$;

(2) for every $\rho>0$, there exists $\Lambda_{\rho}>0$ such that

$$
(\lambda, u) \in \mathcal{C}^{+} \quad \text { with }\|u\|_{\infty}=\rho \quad \Rightarrow \quad \lambda>\Lambda_{\rho} ;
$$

(3) $\mathcal{C}^{-}$is of $\subset$-shaped and joins $(+\infty, \mathbf{0})$ to $(+\infty, \infty)$;

(4) for every $\rho>0$, there exists $\Lambda_{\rho}>0$ such that

$$
(\lambda, u) \in \mathcal{C}^{-} \quad \text { with }\|u\|_{\infty}=\rho \quad \Rightarrow \quad \lambda>\Lambda_{\rho} .
$$

The linear problem

$$
\left\{\begin{array}{l}
u^{\prime \prime \prime \prime}(x)=y(x), \quad x \in(0,1), \\
u(0)=u(1)=u^{\prime \prime}(0)=u^{\prime \prime}(1)=0,
\end{array}\right.
$$


is equivalent to

$$
u(t)=\int_{0}^{1} G(t, s) y(s) d s=: T y(t)
$$

where

$$
G(t, s)=\frac{1}{6} \begin{cases}t(1-s)\left[2 s-s^{2}-t^{2}\right], & 0 \leq t \leq s \leq 1, \\ s(1-t)\left[2 t-t^{2}-s^{2}\right], & 0 \leq s \leq t \leq 1 .\end{cases}
$$

Let

$$
\begin{aligned}
& q(t)=\frac{1}{2} t(1-t), \quad t \in[0,1], \\
& j(s)= \begin{cases}1-\sqrt{\frac{1-s^{2}}{3}}, & s \in[0,1 / 2], \\
\sqrt{\frac{s(2-s)}{3}}, & s \in[1 / 2,1] .\end{cases}
\end{aligned}
$$

Then

$$
\begin{aligned}
& G(j(s), s)=\max _{0 \leq t \leq 1} G(t, s), \\
& G(j(s), s)=\frac{1}{9} \begin{cases}s(1-s)(1+s) \sqrt{\frac{1-s^{2}}{3}}, & s \in[0,1 / 2], \\
s(1-s)(2-s) \sqrt{\frac{s(2-s)}{3}}, & s \in[1 / 2,1],\end{cases} \\
& G(t, s) \geq q(t) G(j(s), s), \quad t \in[0,1] . \\
& G(t, s) \geq \frac{3}{32} G(j(s), s), \quad t \in\left[\frac{1}{4}, \frac{3}{4}\right] .
\end{aligned}
$$

Let

$$
K:=\left\{w \in C[0,1]: \min _{0 \leq t \leq 1} w(t) \geq 0, \min _{1 / 4 \leq t \leq 3 / 4} w(t) \geq \frac{3}{32}\|w\|_{\infty}\right\} .
$$

Corollary 1.1 Let (A1), (A2), (A3), and (A4) hold. Then (1.1) with $h \equiv 1$ has at least two positive solutions and at least two negative solutions (see Fig. 1) provided

$$
\lambda>\hat{\lambda}=:\left(\hat{m}_{\rho} \int_{1 / 4}^{3 / 4} G(j(s), s) h(s) d s\right)^{-1}
$$

where

$$
\hat{m}_{\rho}=\min _{3 \rho / 32 \leq x \leq \rho}\{f(x)\} .
$$

For other related results on the existence and multiplicity of positive solutions and nodal solutions of fourth order problems, see Rynne [13] and Ma [8, 9].

The rest of the paper is arranged as follows: In Sect. 2, we prove some properties of superior limit of certain infinity collection of connected sets. In Sect. 3, we state and prove some properties for the one-sign solutions $(\lambda, u)$ of $(1.1)$. Finally, in Sect. 4, we state and prove our main results. 
Figure 1 Components of one-sign solutions in Theorem 1.1

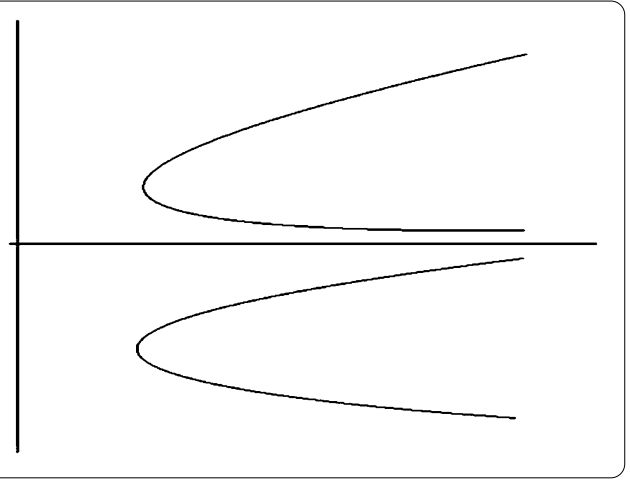

\section{Superior limit and component}

Definition 2.1 ([14]) Let $X$ be a Banach space and $\left\{C_{n} \mid n=1,2, \ldots\right\}$ be a family of subsets of $X$. Then the superior limit $\mathcal{D}$ of $\left\{C_{n}\right\}$ is defined by

$$
\mathcal{D}:=\limsup _{n \rightarrow \infty} C_{n}=\left\{x \in X \mid \exists\left\{n_{i}\right\} \subset \mathbb{N} \text { and } x_{n_{i}} \in C_{n_{i}} \text { such that } x_{n_{i}} \rightarrow x\right\} .
$$

Definition 2.2 ([14]) A component of a set $M$ means a maximal connected subset of $M$.

Lemma 2.1 ([14]) Suppose that $Y$ is a compact metric space, $A$ and $B$ are non-intersecting closed subsets of $Y$, and no component of $Y$ intersects both $A$ and $B$. Then there exist two disjoint compact subsets $Y_{A}$ and $Y_{B}$ such that $Y=Y_{A} \cup Y_{B}, A \subset Y_{A}, B \subset Y_{B}$.

Lemma 2.2 ([11]) Let $X$ be a Banach space and let $\left\{C_{n}\right\}$ be a family of closed connected subsets of $X$. Assume that

(i) there exist $z_{n} \in C_{n}, n=1,2, \ldots$, and $z^{*} \in X$ such that $z_{n} \rightarrow z^{*}$;

(ii) $r_{n}=\sup \left\{\|x\| \mid x \in C_{n}\right\}=\infty$;

(iii) for every $R>0,\left(\bigcup_{n=1}^{\infty} C_{n}\right) \cap B_{R}$ is a relatively compact set of $X$, where

$$
B_{R}=\{x \in X \mid\|x\| \leq R\} .
$$

Then there exists an unbounded component $\mathcal{C}$ in $\mathcal{D}$ and $z^{*} \in \mathcal{C}$.

Let $E=\left\{u \in C^{3}[0,1]: u(0)=u(1)=u^{\prime \prime}(0)=u^{\prime \prime}(1)=0\right\}$ with the norm

$$
\|u\|=\max \left\{\|u\|_{\infty},\left\|u^{\prime}\right\|_{\infty},\left\|u^{\prime \prime}\right\|_{\infty},\left\|u^{\prime \prime \prime}\right\|_{\infty}\right\}
$$

It is well known that the linear eigenvalue problem

$$
\left\{\begin{array}{l}
u^{\prime \prime \prime \prime}=\mu h(x) u(x), \quad x \in(0,1), \\
u(0)=u(1)=u^{\prime \prime}(0)=u^{\prime \prime}(1)=0
\end{array}\right.
$$

has an infinite sequence of simple eigenvalues

$$
0<\mu_{1}<\mu_{2}<\cdots<\mu_{k}<\cdots, \quad k \rightarrow \infty,
$$

and the eigenfunction $\phi_{k}$ corresponding to $\mu_{k}$ has exactly $k-1$ simple zeros in $(0,1)$, see [13]. 


\section{Some preliminary results}

Let us define an operator $T_{\lambda}: Y \rightarrow Y$ by

$$
T_{\lambda} u:=\lambda \int_{0}^{1} G(t, s) h(s) f(u(s)) d s .
$$

Lemma 3.1 Assume that (A1)-(A4) hold. Then $T_{\lambda}: K \rightarrow K$ is completely continuous.

Lemma 3.2 Let $\Omega_{r}:=\left\{u \in K:\|u\|_{\infty}<r\right\}$. Let (A1)-(A4) hold. If $u \in \partial \Omega_{r}, r>0$, then

$$
\left\|T_{\lambda} u\right\|_{\infty} \leq \lambda \hat{M}_{r} \int_{0}^{1} G(j(s), s) h(s) d s,
$$

where $\hat{M}_{r}=\max _{0 \leq s \leq r}\{f(s)\}$.

Proof Since $f(u(t)) \leq \hat{M}_{r}$ for $t \in[0,1]$, it follows that

$$
\begin{aligned}
\left\|T_{\lambda} u\right\|_{\infty} & \leq \lambda \int_{0}^{1} G(j(s), s) h(s) f(u(s)) d s \\
& \leq \lambda \hat{M}_{r} \int_{0}^{1} G(j(s), s) h(s) d s .
\end{aligned}
$$

Lemma 3.3 Let (A1)-(A4) hold. Assume that $\left\{\left(\mu_{k}, y_{k}\right)\right\} \subset(0,+\infty) \times K$ is a sequence of positive solutions of (1.1). Assume that $\mu_{k} \leq C_{0}$ for some constant $C_{0}>0$, and

$$
\lim _{k \rightarrow \infty}\left\|y_{k}^{\prime \prime \prime}\right\|_{\infty}=\infty
$$

Then

$$
\lim _{k \rightarrow \infty}\left\|y_{k}\right\|_{\infty}=\infty
$$

Proof Assume on the contrary that $\left\{\left\|y_{k}\right\|_{\infty}\right\}$ is bounded. Then

$$
\left\|\mu_{k} h(x) f\left(y_{k}(x)\right)\right\|_{\infty} \leq M
$$

for some constant $M$ that is independent of $k$. Thus, it follows from the relation

$$
y_{k}^{\prime \prime \prime \prime}(x)=\mu_{k} h(x) f\left(y_{k}(x)\right)
$$

that $\left\{y_{k}^{\prime \prime \prime \prime}\right\}$ is uniformly bounded in $C[0,1]$, and subsequently $\left\{y_{k}^{\prime \prime \prime}\right\}$ is uniformly bounded in $C[0,1]$. However, this contradicts (3.2).

Lemma 3.4 Assume that (A1)-(A4) hold. If $u \in \partial \Omega_{r}, r>0$, then

$$
\left\|T_{\lambda} u\right\|_{\infty} \geq \lambda \hat{m}_{r} \int_{1 / 4}^{3 / 4} G(j(s), s) h(s) d s
$$

where

$$
\hat{m}_{r}=\min _{3 r / 32 \leq x \leq r}\{f(x)\} .
$$


Proof Since $f(u(t)) \geq \hat{m}_{r}$ for $t \in\left[\frac{1}{4}, \frac{3}{4}\right]$, it follows that

$$
\begin{aligned}
\left\|T_{\lambda} u\right\|_{\infty} & \geq \lambda \int_{0}^{1} G(j(s), s) h(s) f(u(s)) d s \\
& \geq \lambda \hat{m}_{r} \int_{1 / 4}^{3 / 4} G(j(s), s) h(s) d s .
\end{aligned}
$$

\section{Proof of the main results}

We only deal with the global behavior of positive solutions of (1.1). The global behavior of negative solutions of (1.1) can be treated by a similar method.

Let $\Sigma^{+}$be the closure of the set of positive solutions of (1.1) in $E$. To prove Theorem 1.1, we will develop a bifurcation approach to treat the case $f_{0}=0$. Crucial to this approach is to construct a sequence of functions $\left\{f^{[n]}\right\}$ that is asymptotic linear at 0 and satisfies

$$
f^{[n]} \rightarrow f, \quad\left(f^{[n]}\right)_{0} \rightarrow 0
$$

By means of the corresponding auxiliary equations, we obtain a sequence of unbounded components $\left\{C_{+}^{[n]}\right\}$ via nonlinear Krein-Rutman bifurcation theorem, see Dancer [3] and Zeidler [15], and this enables us to find unbounded components $\hat{\zeta}$ satisfying

$$
\hat{\zeta} \subset \limsup _{n \rightarrow \infty} C_{+}^{[n]}
$$

and joining $(+\infty, 0)$ with $(+\infty, \infty)$.

Define $g^{[n]}: \mathbb{R} \rightarrow \mathbb{R}$ by

$$
g^{[n]}(s)= \begin{cases}f(s), & s \in\left(\frac{1}{n}, \infty\right) \cup\left(-\infty,-\frac{1}{n}\right), \\ n f\left(\frac{1}{n}\right) s, & s \in\left[-\frac{1}{n}, \frac{1}{n}\right]\end{cases}
$$

Then $g^{[n]} \in C(\mathbb{R}, \mathbb{R})$ with

$$
s g^{[n]}(s)>0, \quad \forall|s| \in(0, \infty), \quad \text { and } \quad\left(g^{[n]}\right)_{0}=n f\left(\frac{1}{n}\right)
$$

By (A3), it follows that

$$
\lim _{n \rightarrow \infty}\left(g^{[n]}\right)_{0}=0
$$

To apply the nonlinear Krein-Rutman theorem [4], let us consider the auxiliary family of the equations

$$
\begin{aligned}
& u^{\prime \prime \prime \prime}=\lambda h(t) g^{[n]}(u), \quad t \in(0,1), \\
& u(0)=u(1)=u^{\prime \prime}(0)=u^{\prime \prime}(1)=0 .
\end{aligned}
$$

Let $\xi^{[n]} \in C(\mathbb{R})$ be such that

$$
g^{[n]}(u)=\left(g^{[n]}\right)_{0} u+\xi^{[n]}(u)=n f\left(\frac{1}{n}\right) u+\xi^{[n]}(u) .
$$


Then

$$
\lim _{|u| \rightarrow 0} \frac{\xi^{[n]}(u)}{u}=0
$$

Let $D:=\left\{u \in C^{4}[0,1]: u(0)=u(1)=u^{\prime \prime}(0)=u^{\prime \prime}(1)=0\right\}$. Let $L: D \rightarrow Y$ be the linear operator defined by

$$
L u:=u^{\prime \prime \prime \prime}, \quad u \in D .
$$

Let us consider

$$
L u-\lambda h(t)\left(g^{[n]}\right)_{0} u=\lambda h(t) \xi^{[n]}(u)
$$

as a bifurcation problem from the trivial solution $u \equiv 0$.

Equation (4.7) can be converted to the equivalent equation

$$
\begin{aligned}
u(t) & =\int_{0}^{1} G(t, s)\left[\lambda h(s)\left(g^{[n]}\right)_{0} u(s)+\lambda h(s) \xi^{[n]}(u(s))\right] d s \\
& :=\lambda L^{-1}\left[h(\cdot)\left(g^{[n]}\right)_{0} u(\cdot)\right](t)+\lambda L^{-1}\left[h(\cdot) \xi^{[n]}(u(\cdot))\right](t) .
\end{aligned}
$$

Further we note that $\left\|L^{-1}\left[h(\cdot) \xi^{[n]}(u(\cdot))\right]\right\|_{\infty}=o\left(\|u\|_{\infty}\right)$ for $u$ near $\theta$ in $E$.

By the fact $\left(g^{[n]}\right)_{0}>0$, the results of nonlinear Krein-Rutman theorem (see Dancer [3] and Zeidler [15, Corollary 15.12]) for (4.7) can be stated as follows: there exists a continuum $C_{+}^{[n]}$ of positive solutions of (4.7) joining $\left(\frac{\lambda_{1}}{\left(g^{[n]}\right)_{0}}, \theta\right)$ to infinity in $[0, \infty) \times K$. Moreover, $C_{+}^{[n]} \backslash\left\{\left(\frac{\lambda_{1}}{\left(g^{[n]}\right)_{0}}, \theta\right)\right\} \subset([0, \infty) \times \operatorname{int} K)$ and $\left(\frac{\lambda_{1}}{\left(g^{[n]}\right)_{0}}, \theta\right)$ is the only positive bifurcation point of (4.7) lying on the trivial solutions line $u \equiv \theta$.

Lemma 4.1 Let (A1)-(A4) hold. Then, for each fixed $n, C_{+}^{[n]}$ joins $\left(\frac{\lambda_{1}}{\left(g^{[n]}\right)_{0}}, \theta\right)$ to $(\infty, \infty)$ in $[0, \infty) \times K$.

Proof We divide the proof into two steps.

Step 1. We show that $\sup \left\{\lambda \mid(\lambda, u) \in C_{+}^{[n]}\right\}=\infty$.

Assume on the contrary that $\sup \left\{\lambda \mid(\lambda, u) \in C_{+}^{[n]}\right\}=: c_{0}<\infty$. Let $\left\{\left(\mu_{k}, y_{k}\right)\right\} \subset C_{+}^{[n]}$ be such that

$$
\left|\mu_{k}\right|+\left\|y_{k}\right\|_{\infty} \rightarrow \infty
$$

Then $\left\|y_{k}\right\|_{\infty} \rightarrow \infty$. This together with the fact

$$
\min _{\sigma \leq t \leq 1-\sigma} y_{k}(t) \geq q(\sigma)\left\|y_{k}\right\|_{\infty}, \quad \forall 0<\sigma<\frac{1}{2}
$$

implies that, for arbitrary $\sigma \in\left(0, \frac{1}{2}\right)$,

$$
\lim _{k \rightarrow \infty} y_{k}(t)=\infty, \quad \text { uniformly for } t \in[\sigma, 1-\sigma] \text {. }
$$


Since $\left(\mu_{k}, y_{k}\right) \in C_{+}^{[n]}$, we have that

$$
\begin{aligned}
& y_{k}^{\prime \prime \prime \prime}(t)=\mu_{k} h(t) g^{[n]}\left(y_{k}(t)\right), \quad t \in(0,1), \\
& y_{k}(0)=y_{k}(1)=y_{k}^{\prime \prime}(0)=y_{k}^{\prime \prime}(1)=0 .
\end{aligned}
$$

Set $v_{k}(t)=\frac{y_{k}(t)}{\left\|y_{k}\right\|_{\infty}}$. Then

$$
\begin{aligned}
& \left\|v_{k}\right\|_{\infty}=1, \\
& v_{k}^{\prime \prime \prime \prime}(t)=\mu_{k} h(t) \frac{g^{[n]}\left(y_{k}(t)\right)}{y_{k}(t)} v_{k}(t), \quad t \in(0,1), \\
& v_{k}(0)=v_{k}(1)=v_{k}^{\prime \prime}(0)=v_{k}^{\prime \prime}(1)=0 .
\end{aligned}
$$

From (4.13) and the fact that $\left(g^{[n]}\right)_{\infty}=0$, we conclude that

$$
\left\|v_{k}^{\prime \prime \prime \prime}\right\|_{\infty} \leq M
$$

for some constant $M>0$ independent of $k$.

Now, choosing a subsequence and relabeling if necessary, it follows that there exists $\left(\mu_{*}, v_{*}\right) \in\left[0, c_{0}\right] \times E$ with

$$
\left\|v_{*}\right\|_{\infty}=1
$$

such that

$$
\lim _{k \rightarrow \infty}\left(\mu_{k}, v_{k}\right)=\left(\mu_{*}, v_{*}\right), \quad \text { in }\left[0, c_{0}\right] \times E
$$

Notice that (4.13), (4.14) is equivalent to

$$
v_{k}(t)=\mu_{k} \int_{0}^{1} G(t, s) h(s) \frac{g^{[n]}\left(y_{k}(s)\right)}{y_{k}(s)} v_{k}(s) d s, \quad t \in(0,1) .
$$

Combining this with (4.16) and using (4.10) and the Lebesgue dominated convergence theorem, we conclude that

$$
\nu_{*}(t)=\mu_{*} \int_{0}^{1} G(t, s) h(s) 0 \nu_{*}(s) d s=0, \quad t \in(0,1) .
$$

This contradicts (4.15). Therefore

$$
\sup \left\{\lambda \mid(\lambda, y) \in C_{+}^{[n]}\right\}=\infty
$$

Step 2. We show that $\sup \left\{\|u\|_{\infty} \mid(\lambda, u) \in C_{+}^{[n]}\right\}=\infty$.

Assume on the contrary that $\sup \left\{\|u\|_{\infty} \mid(\lambda, u) \in C_{+}^{[n]}\right\}=: M_{\infty}<\infty$. Let $\left\{\left(\mu_{k}, y_{k}\right)\right\} \subset C_{+}^{[n]}$ be such that

$$
\mu_{k} \rightarrow \infty, \quad\left\|y_{k}\right\|_{\infty} \leq M_{\infty} .
$$


Since $\left(\mu_{k}, y_{k}\right) \in C_{+}^{[n]}$, for any $t \in[\sigma, 1-\sigma]$, we have from (1.2) that

$$
\begin{aligned}
y_{k}(t) & =\mu_{k} \int_{0}^{1} G(t, s) h(s) g^{[n]}\left(y_{k}(s)\right) d s \\
& \geq \mu_{k} \int_{\sigma}^{1-\sigma} q(\sigma) G(j(s), s) h(s) \frac{g^{[n]}\left(y_{k}(s)\right)}{y_{k}(s)} y_{k}(s) d s \\
& \geq \mu_{k} \int_{\sigma}^{1-\sigma} q(\sigma) G(j(s), s) h(s) \frac{g^{[n]}\left(y_{k}(s)\right)}{y_{k}(s)} q(\sigma) d s\left\|_{k}\right\|_{\infty} \\
& \geq \mu_{k} \int_{\sigma}^{1-\sigma} q^{2}(\sigma) G(j(s), s) h(s) b_{*} d s\left\|y_{k}\right\|_{\infty}
\end{aligned}
$$

(where $\left.b_{*}:=\inf \left\{\frac{g^{[n]}(x)}{x} \mid x \in\left(0, M_{\infty}\right]\right\}>0\right)$, which yields that $\left\{\mu_{k}\right\}$ is bounded. However, this contradicts (4.17).

Therefore, $C_{+}^{[n]}$ joins $\left(\frac{\lambda_{1}}{\left(g^{[n]}\right)_{0}}, \theta\right)$ to $(\infty, \infty)$ in $K$.

Lemma 4.2 Let (A1)-(A4) hold and let $I \subset(0, \infty)$ be a closed interval. Then there exists a positive constant $M$ such that

$$
\sup \left\{\|y\|_{\infty} \mid(\mu, y) \in C_{+}^{[n]} \text { and } \mu \in I\right\} \leq M
$$

Proof Assume on the contrary that there exists a sequence $\left\{\left(\mu_{k}, y_{k}\right)\right\} \subset C_{+}^{[n]} \cap(I \times K)$ such that

$$
\left\|y_{k}\right\|_{\infty} \rightarrow \infty
$$

Then, (4.9), (4.10), (4.11), and (4.12) hold. Set $v_{k}(t)=\frac{y_{k}(t)}{\left\|y_{k}\right\|_{\infty}}$. Then

$$
\left\|v_{k}\right\|_{\infty}=1
$$

Now, choosing a subsequence and relabeling if necessary, it follows that there exists $\left(\mu_{*}, \nu_{*}\right) \in I \times Y$ with

$$
\left\|v_{*}\right\|_{\infty}=1
$$

such that

$$
\lim _{k \rightarrow \infty}\left(\mu_{k}, v_{k}\right)=\left(\mu_{*}, v_{*}\right) \quad \text { in } \mathbb{R} \times Y .
$$

Moreover, from (4.11), (4.12), (4.10) and the assumption $f_{\infty}=0$, it follows that

$$
\begin{aligned}
& v_{*}^{\prime \prime \prime \prime}(t)=\mu_{*} h(t) \cdot 0, \quad t \in(0,1), \\
& v_{*}(0)=v_{*}(1)=v_{*}^{\prime \prime}(0)=v_{*}^{\prime \prime}(1)=0,
\end{aligned}
$$

and subsequently, $v_{*}(t) \equiv 0$ for $t \in[0,1]$. This contradicts (4.18). Therefore

$$
\sup \left\{\|y\|_{\infty} \mid(\mu, y) \in C_{+}^{[n]} \text { and } \mu \in I\right\} \leq M
$$


Lemma 4.3 Let (A1)-(A4) hold. Then there exists $\rho^{*}>0$ such that

$$
\left(\bigcup_{n=1}^{\infty} C_{+}^{[n]}\right) \cap\left(\left(0, \rho^{*}\right) \times K\right)=\emptyset .
$$

Proof Assume on the contrary that there exists $\left\{\left(\mu_{k}, y_{k}\right)\right\} \subset\left(\bigcup_{n=1}^{\infty} C_{+}^{[n]}\right) \cap((0,+\infty) \times K)$ such that $\mu_{k} \rightarrow 0$. Then

$$
y_{k}(t)=\mu_{k} \int_{0}^{1} G(t, s) h(s) g^{[n]}\left(y_{k}(s)\right) d s, \quad t \in(0,1) .
$$

Set $v_{k}(t)=\frac{y_{k}(t)}{\left\|y_{k}\right\|_{\infty}}$. Then

$$
\left\|v_{k}\right\|_{\infty}=1
$$

and for all $t \in(0,1)$,

$$
\begin{aligned}
v_{k}(t) & =\mu_{k} \int_{0}^{1} G(t, s) h(s) \frac{g^{[n]}\left(y_{k}(s)\right)}{y_{k}(s)} \frac{y_{k}(s)}{\left\|y_{k}\right\|_{\infty}} d s \\
& \leq \mu_{k} \int_{0}^{1} G(j(s), s) h(s) B_{n}^{*}\left\|v_{k}\right\|_{\infty} d s,
\end{aligned}
$$

where $B_{n}^{*}=\sup \left\{\frac{g^{[n]}(x)}{x} \mid x \in(0, \infty), n \in \mathbb{N}\right\}$. Let

$$
B^{*}=\sup \left\{B_{n}^{*} \mid n \in \mathbb{N}\right\} \text {. }
$$

Then $B^{*}<\infty$, and

$$
v_{k}(t) \leq \mu_{k} \int_{0}^{1} G(j(s), s) h(s) B^{*}\left\|v_{k}\right\|_{\infty} d s \rightarrow 0,
$$

which contradicts the fact $\left\|v_{k}\right\|_{\infty}=1$. Therefore, there exists $\rho^{*}>0$, such that

$$
\left(\bigcup_{n=1}^{\infty} C_{+}^{[n]}\right) \cap\left(\left(0, \rho^{*}\right) \times K\right)=\emptyset .
$$

Proof of Theorem 1.1 By Lemmas 4.1-4.3 and the similar method to prove Ma and An [12, Theorem 4.1], with obvious changes, we may get a desired connected component $\mathcal{C}^{+} \subset$ $\lim \sup C_{+}^{[n]}$ of positive solutions of (1.1) and a connected component $\mathcal{C}^{-} \subset \lim \sup C_{-}^{[n]}$ of negative solutions of (1.1) such that

(1) $\mathcal{C}^{+}$is of $\subset$-shaped and joins $(+\infty, \theta)$ to $(+\infty, \infty)$;

(2) for every $\rho>0$, there exists $\Lambda_{\rho}>0$ such that

$$
(\lambda, u) \in \mathcal{C}^{+} \quad \text { with }\|u\|_{\infty}=\rho \quad \Rightarrow \quad \lambda>\Lambda_{\rho}
$$

(3) $\mathcal{C}^{-}$is of $\subset$-shaped and joins $(+\infty, \theta)$ to $(+\infty, \infty)$;

(4) for every $\rho>0$, there exists $\Lambda_{\rho}>0$ such that

$$
(\lambda, u) \in \mathcal{C}^{-} \quad \text { with }\|u\|_{\infty}=\rho \quad \Rightarrow \quad \lambda>\Lambda_{\rho} .
$$


Acknowledgements

The authors are very grateful to an anonymous referee for his or her very valuable suggestions.

\section{Funding}

This work was supported by the National Natural Science Foundation of China (No. 11671322).

\section{Abbreviations}

Not applicable.

Availability of data and materials

Data sharing not applicable to this article as no datasets were generated.

\section{Competing interests}

All of the authors of this article claim that together they have no competing interests.

\section{Authors' contributions}

The authors claim that the research was realized in collaboration with the same responsibility. All authors read and approved the last version of the manuscript.

\section{Publisher's Note}

Springer Nature remains neutral with regard to jurisdictional claims in published maps and institutional affiliations.

Received: 22 January 2020 Accepted: 13 April 2020 Published online: 25 April 2020

\section{References}

1. Bonanno, G., Di Bella, B.: A boundary value problem for fourth-order elastic beam equations. J. Math. Anal. Appl. 343, 1166-1176 (2008)

2. Cabada, A., Enguiça, R.R.: Positivity and lower and upper solutions for fourth order boundary value problems. Nonlinear Anal. 67(5), 1599-1612 (2007)

3. Dancer, E.: Global solutions branches for positive maps. Arch. Ration. Mech. Anal. 55, 207-213 (1974)

4. Deimling, K.: Nonlinear Functional Analysis. Springer, Berlin (1985)

5. Drábek, P., Holubová, G.: On the maximum and antimaximum principles for the beam equation. Appl. Math. Lett. 56 29-33 (2016)

6. Jiang, D., Liu, H., Xu, X.: Nonresonant singular fourth-order boundary value problems. Appl. Math. Lett. 18(1), 69-75 (2005)

7. Li, Y:: On the existence of positive solutions for the bending elastic beam equations. Appl. Math. Comput. 189(1), $821-827$ (2007)

8. Ma, R.: Existence of positive solutions of a fourth-order boundary value problem. Appl. Math. Comput. 168(2) 1219-1231 (2005)

9. Ma, R.: Nodal solutions of boundary value problems of fourth-order ordinary differential equations. J. Math. Anal. Appl. 319(2), 424-434 (2006)

10. Ma, R.: Nodal solutions for a fourth-order two-point boundary value problem. J. Math. Anal. Appl. 314(1), 254-265 (2006)

11. Ma, R., An, Y.: Global structure of positive solutions for superlinear second order m-point boundary value problems. Topol. Methods Nonlinear Anal. 34(2), 279-290 (2009)

12. Ma, R., An, Y.: Global structure of positive solutions for nonlocal boundary value problems involving integral conditions. Nonlinear Anal. 71, 4364-4376 (2009)

13. Rynne, B.P.: Infinitely many solutions of superlinear fourth order boundary value problems. Topol. Methods Nonlinear Anal. 19(2), 303-312 (2002)

14. Whyburn, G.T.: Topological Analysis. Princeton University Press, Princeton (1958)

15. Zeidler, E.: Nonlinear Functional Analysis and Its Applications. Springer, New York (1986)

\section{Submit your manuscript to a SpringerOpen ${ }^{\circ}$ journal and benefit from:}

- Convenient online submission

- Rigorous peer review

- Open access: articles freely available online

- High visibility within the field

- Retaining the copyright to your article

Submit your next manuscript at $\boldsymbol{~ s p r i n g e r o p e n . c o m ~}$ 